\title{
$\delta$ Scuti-type pulsation in the hot component of the Algol-type binary system BG Peg
}

\author{
T. Şenyüz ${ }^{1}$ and E. Soydugan ${ }^{1,2}$ \\ ${ }^{1}$ Astrophysics Research Centre and Observatory, Çanakkale Onsekiz Mart University, \\ Çanakkale, Turkey \\ email: tuncsenyuz@gmail.com \\ ${ }^{2}$ Department of Physics, Faculty of Arts and Sciences, \\ Çanakkale Onsekiz Mart University, Çanakkale, Turkey \\ email: esoydugan@comu.edu.tr
}

\begin{abstract}
In this study, 23 Algol-type binary systems, which were selected as candidate binaries with pulsating components, were observed at the Çanakkale Onsekiz Mart University Observatory. One of these systems was BG Peg. Its hotter component shows $\delta$ Scuti-type light variations. Physical parameters of BG Peg were derived from modelling the $V$ light curve using the Wilson-Devinney code. The frequency analysis shows that the pulsational component of the BG Peg system pulsates in two modes with periods of 0.039 and $0.047 \mathrm{~d}$. Mode identification indicates that both modes are most likely non-radial $l=2$ modes.
\end{abstract}

Keywords. techniques: photometric, stars: binaries: eclipsing, stars: individual (BG Peg)

\section{Introduction}

Algol-type eclipsing binary system BG Peg $\left(V=10.50 \mathrm{mag}, \mathrm{P}_{\text {orb }}=1.952443 \mathrm{~d}\right)$ was listed in the catalogue of Algol-type binary stars (Svechnikov \& Kuznetsova 1990). It was also listed by Soydugan et al. (2006) as a system with the component in the $\delta$ Sct instability strip. Soydugan et al. (2009) discovered $\delta$ Scuti-type light variations in the hotter component of the system. The $\delta$ Scuti-type variability in BG Peg was also found independently by Dvorak (2009). In this paper, photometric study of BG Peg made using only $V$-filter data are presented.

\section{Photometric observations}

BG Peg was photometrically observed in Johnson's $B$ and $V$ filters over 13 nights during 2008 observing season at the Çanakkale Onsekiz Mart University Observatory using the 40-cm Schmidt-Cassegrain telescope equipped with a SBIG STL-1001E CCD camera. TYC 1698.1052 and TYC 1698.1142 were selected as comparison and check stars, respectively. The standard error of this photometry was about 0.010 mag in both filters.

\section{Light curve and frequency analysis of BG Peg}

The light curve of BG Peg was analyzed using the Wilson-Devinney (WD) program in a semi-detached configuration. From the analysis of the light curve, we derived the following parameters: phase shift, orbital inclination, dimensionless potential of the primary component, mass ratio and fractional luminosity of the primary component. The other parameters were kept fixed. The temperature of the primary was adopted following Popper (1980), namely $8770 \mathrm{~K}$ for A2 spectral type. Our WD solution is the following: $i=83.2^{\circ}, T_{\text {eff }, 2}=5155 \mathrm{~K}, \Omega_{1}=3.628, \Omega_{2}=2.313, q=0.233, r_{1, \text { mean }}=0.296$, and 


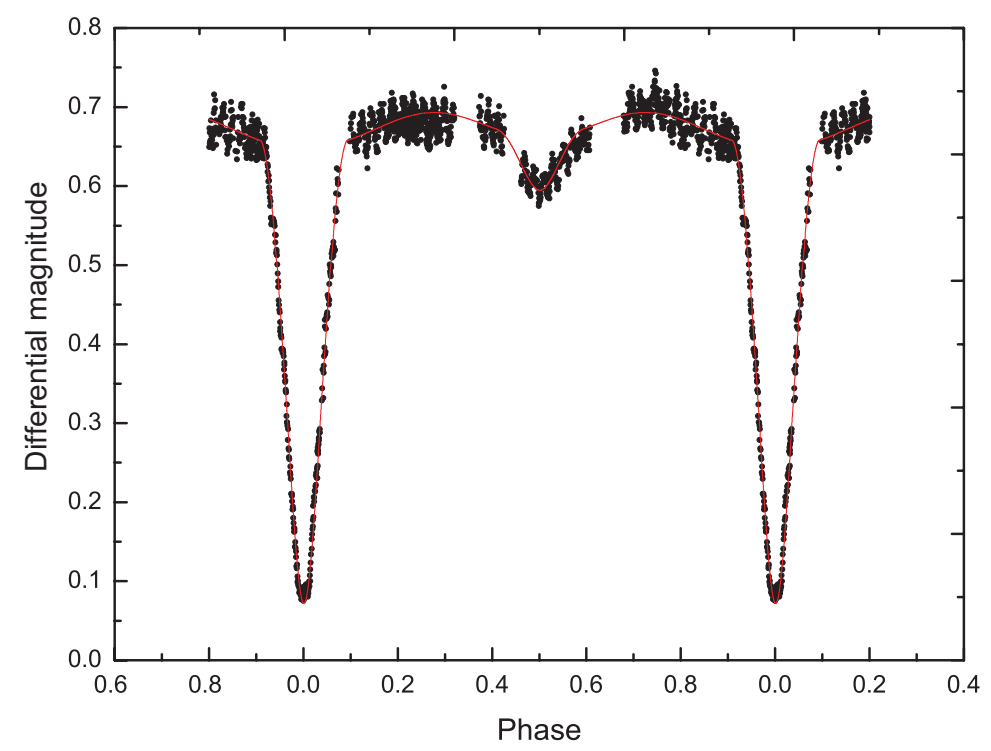

Figure 1. Synthetic (continuous line) and observed (dots) light curve of BG Peg in $V$ filter.

$r_{2, \text { mean }}=0.265$. The comparison of the theoretical and observed light curves is presented in Fig. 1.

A frequency analysis of the $V$-filter data was made using PERIOD 04 program (Lenz \& Breger 2005). Pulsational frequencies of the primary component were found to be equal to $25.54 \mathrm{~d}^{-1}$ and $21.05 \mathrm{~d}^{-1}$. The pulsation amplitudes in $V$ filter we determined are equal to 0.0153 and $0.0072 \mathrm{mag}$ for the two modes, respectively.

\section{Conclusion}

Stars, which were selected from the catalogue of eclipsing binaries with candidate $\delta$ Scuti-type components prepared by Soydugan et al. (2006), have been observed since 2007. We discovered $\delta$ Scuti-type pulsations in the hotter components of BG Peg, DY Aqr and IO UMa. Two pulsational frequencies were detected in the hotter component of BG Peg. Using the FAMIAS program, we obtained spherical harmonic degrees to be $l=2$ for both modes observed in this star.

\section{Acknowledgement}

We wish to thank the Turkish Scientific and Technical Research Council (Grant no. 107T634) for supporting this study.

\section{References}

Dvorak, S. 2009, CoAst, 160, 64

Lenz, P. \& Breger, M. 2005, CoAst, 146, 53

Popper, D. M. 1980, ARAA, 18, 115

Soydugan, E., Soydugan, F., \& Demircan, O., İbanoğlu, C. 2006, MNRAS, 370, 2013

Soydugan, E., Soydugan, F., Şenyüz, T., et al. 2009, IBVS, 5902

Svechnikov, M. A. \& Kuznetsova, E. F. 1990, Catalogue of Approximate Photometric and Absolute Elements of Eclipsing Variable Stars (Izd-vo Ural'skogo universiteta)

Wilson, R. E. \& Devinney, R. J. 1971, ApJ, 166, 605

Zima, W. 2008, CoAst, 155, 17 